\title{
ConvergenceConcepts: An R Package to Investigate Various Modes of Convergence
}

\author{
by Pierre Lafaye de Micheaux and Benoit Liquet
}

\begin{abstract}
ConvergenceConcepts is an R package, built upon the tkrplot, tcltk and lattice packages, designed to investigate the convergence of simulated sequences of random variables. Four classical modes of convergence may be studied, namely: almost sure convergence (a.s.), convergence in probability $(P)$, convergence in law $(L)$ and convergence in $r$-th mean $(r)$. This investigation is performed through accurate graphical representations. This package may be used as a pedagogical tool. It may give students a better understanding of these notions and help them to visualize these difficult theoretical concepts. Moreover, some scholars could gain some insight into the behaviour of some random sequences they are interested in.
\end{abstract}

\section{Introduction}

Many students are exposed, during their graduate school years, to the difficult concepts of convergence of a sequence of random variables (see Sethuraman (1995)). Indeed, as pointed out by Bryce, Gould, Notz and Peck (2001), "statistical theory is an important part of the curriculum, and is particularly important for students headed for graduate school". Such knowledge is prescribed by learned statistics societies (see Accreditation of Statisticians by the Statistical Society of Canada, and Curriculum Guidelines for Undergraduate Programs in Statistical Science by the American Statistical Association). In the main textbooks (see for example Billingsley (1986), Chung (1974), Ferguson (1996), Lehmann (2001), Serfling (2002)), around 15 pages without graphs are allotted to defining these convergence concepts and their interrelations. But, very often, these concepts are only described through their definitions and some of their properties. Thus, some students may not fully visualize how a random variable converges to some limit. They also may not fully understand the differences between the various modes, especially between convergence in probability and almost surely.

Moreover, a statistician could be interested in whether or not a specific random sequence converges. To explain the modes of convergence, we could follow Bryce, Gould, Notz and Peck (2001)'s advice: "a modern statistical theory course might, for example, include more work on computer intensive methods". With regard to the convergence in law, Dunn (1999) and Marasinghe, Meeker, Cook and Shin (1996) have proposed tools to explain this concept in an interactive manner. Mills (2002) proposed a review of statistical teaching based on simulation methods and Chance and Rossman (2006) have written a book on this subject. Our package enables one to investigate graphically the four classical modes of convergence of a sequence of random variables: convergence almost surely, convergence in probability, convergence in law and convergence in $r$-th mean. Note that it is tightly associated with the reading of Lafaye de Micheaux and Liquet (2009) which explains what we call our "mind visualization approach" of these convergence concepts.

The two main functions to use in our package are investigate and check. convergence. The first one will be described in the next section, investigating pre-defined Exercise 1 from Lafaye de Micheaux and Liquet (2009). The second one will be described in the last section, where it is shown how to treat your own examples.

At this point, note the necessary first two steps to perform before working with our package:

install.packages ( "ConvergenceConcepts")

require (ConvergenceConcepts)

\section{Pre-defined examples}

Our package contains several pre-defined examples and exercises (all introduced and solved in Lafaye de Micheaux and Liquet (2009) and in its associated online Appendix), some of which are classical ones. To investigate these examples, just type in the R console:

investigate()

Any entry can be selected by clicking in the left panel displayed in Figure 1. The corresponding text then appears inside the right panel. Next, by clicking the $\mathrm{OK}$ button, the relevant $\mathrm{R}$ functions are called to help the user to visualize the chosen modes of convergence for the random variable sequence under investigation. You will then be able to twiddle a few features.

For example, the first entry corresponds to the following problem.

Exercise 1: Let $Z$ be a uniform $U[0,1]$ random variable and define $X_{n}=1_{\left[m / 2^{k},(m+1) / 2^{k}\right]}(Z)$ where $n=$ $2^{k}+m$ for $k \geq 1$ and with $0 \leq m<2^{k}$. Thus $X_{1}=1$, $X_{2}=1_{[0,1 / 2)}(Z), X_{3}=1_{[1 / 2,1)}(Z), X_{4}=1_{[0,1 / 4)}(Z)$, $X_{5}=1_{[1 / 4,1 / 2)}(Z), \ldots$ Does $X_{n} \stackrel{\text { a.s. }}{\longrightarrow} 0$ ? Does $X_{n} \stackrel{P}{\longrightarrow}$ $0 ?$

Solution to exercise 1: The drawing on Figure 2 explains the construction of $X_{n}$. 


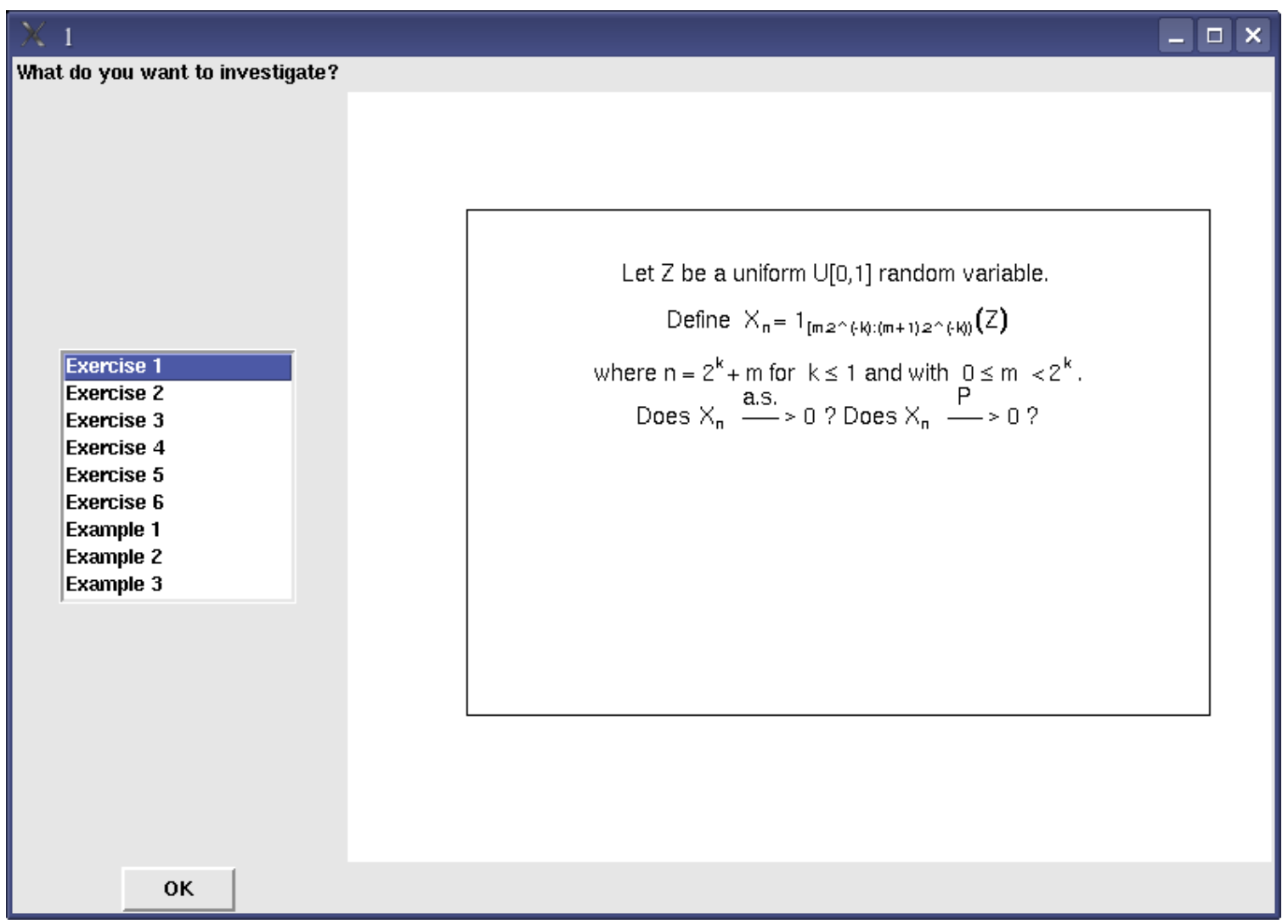

Figure 1: A call to investigate().

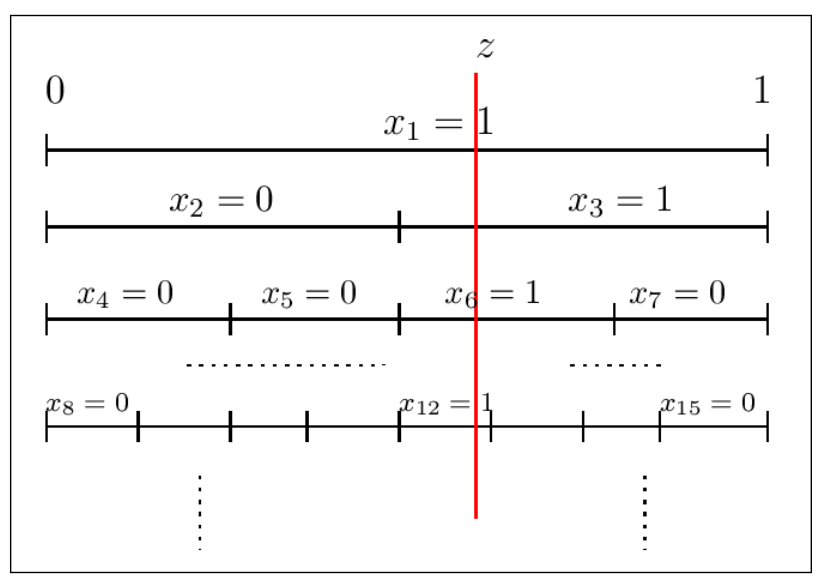

Figure 2: One fictitious sample path for $X_{n}$.

Let us apply our visual reasoning as explained in Section 2 of (Lafaye de Micheaux and Liquet, 2009). Once a $z$ value is randomly drawn, the entire associated sample path is fully determined. As $n$ increases, each sample path "stays" for a longer time at 0 but eventually jumps to 1 . In fact it will jump to 1 an infinite number of times after each fixed $n$ value.

This can be seen using our package. After having selected the first entry and clicked on the OK button displayed in Figure 1, we obtain the following plot.

On the left panel of this plot, we see some sample paths associated with $X_{n}$. One can thus notice, on left panel of Figure 3 , that for all $n=1, \ldots$, all these sample paths will jump to 1 somewhere (and even at many places) in the grey block beginning at position $n$.

By definition, $X_{n} \stackrel{\text { a.s. }}{\longrightarrow} 0$ if and only if $\forall \epsilon>0: a_{n}=$ $\mathbb{P}\left[\omega ; \exists k \geq n:\left|X_{k, \omega}\right|>\epsilon\right] \underset{n \rightarrow \infty}{\longrightarrow} 0$. In this definition, and in the one thereafter on convergence in probability, $\omega$ can be viewed as some kind of labelling of a sample path. We define $\hat{a}_{n}$ to be a frequentist estimate of $a_{n}$. It is the proportion of the pieces of generated sample paths beginning at position $n$ that go outside the horizontal band $[-\epsilon,+\epsilon]$ (see Lafaye de Micheaux and Liquet (2009) for more details). Using our package, the user can interactively move the grey block on left side of Figure 3. He can thus observe the pieces of sample paths which leave the horizontal band. Red marks indicate, for each sample path, the first time when this happens. Simultaneously we can observe their proportion $\hat{a}_{n}$ (equal to 1 here) on right side of Figure 3 as indicated by a sliding red circle. We can see that we cannot have almost sure convergence.

By definition, $X_{n} \stackrel{P}{\longrightarrow} 0$ if and only if $\forall \epsilon>0: p_{n}=$ $\mathbb{P}\left[\omega ;\left|X_{n, \omega}\right|>\epsilon\right] \underset{n \rightarrow \infty}{\longrightarrow} 0$. We define $\hat{p}_{n}$ to be a frequentist estimate of $p_{n}$. It is the proportion of generated sample paths lying outside a band $[-\epsilon,+\epsilon]$ in the bar at position $n$. Note that, for this example, this corresponds to the proportion of $[0,1]$-uniform $z$ values falling into an interval whose length gets narrower. We can investigate graphically convergence in prob- 


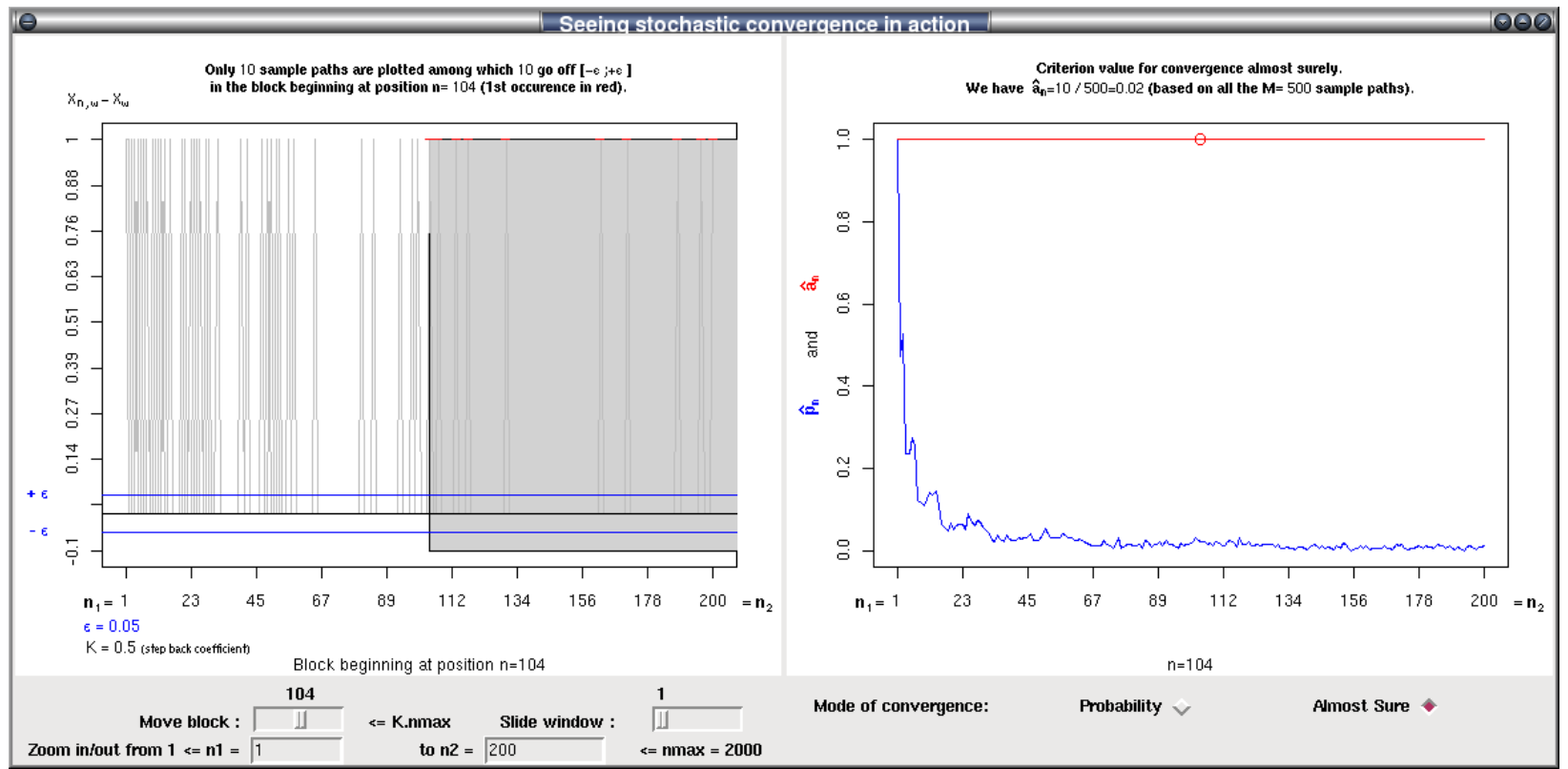

Figure 3: Seeing almost sure convergence in action. On the left panel, we visualize only 10 sample paths among the $M=500$ simulated realizations of the sequence of random variables defined in exercise 1 . It can be seen that all these sample paths go off $[-\epsilon,+\epsilon]$ in the block beginning at position $n=104$. On the right panel, we visualize the $\hat{a}_{n}$ values for each $n$ between 1 and 200. We see that $\hat{a}_{n}$ equal 1 .

ability by sliding the vertical bar (click first on radio button Probability for this bar to appear, see Figure 4) and observe that $\hat{p}_{n}$ is going towards 0 . This lets us perceive that in this case, we do have convergence in probability.

For the interested reader, a mathematically rigorous proof of this exercise can be found in Lafaye de Micheaux and Liquet (2009).

\section{Investigating your own examples}

We now introduce two new problems that have not been either pre-included or treated in the package. We show how a user can define his own functions in order to investigate the convergence of $X_{n}$ towards $X$, or equivalently of $X_{n}-X$ to 0 . These problems are rather simple, but the objective here is only to show how to use our package. The two steps will consist in coding your generator of the $X_{i}$ 's and then using the check. convergence function.

This last function has several arguments whose description is now given.

nmax: number of points in each sample path.

$M$ : number of sample paths to be generated.

gen $X_{n}$ : a function that generates the first $n X_{n}-X$ values, or only the first $n X_{n}$ values in the law case.

$\operatorname{args} X_{n}$ : a list of arguments to gen $X_{n}$.

mode: a character string specifying the mode of convergence to be investigated, must be one of "p" (default), "as", "r" or "L". epsilon: a numeric value giving the interval endpoint.

$r$ : a numeric value $(r>0)$ if convergence in $r$-th mean is to be studied.

nb.sp: number of sample paths to be drawn on the left plot.

density: if density=TRUE, then the plot of the density of $X$ and the histogram of $X_{n}$ is returned. If density $=$ FALSE, then the plot of the distribution function $F(t)$ of $X$ and the empirical distribution $F_{n}(t)$ of $X_{n}$ is returned.

densfunc: function to compute the density of $X$.

probfunc: function to compute the distribution function of $X$.

tinf: lower limit for investigating convergence in law. tsup: upper limit for investigating convergence in law.

trace: function used to draw the plot; plot or points. ...: optional arguments to trace.

Problem 1: Let $X_{1}, X_{2}, \ldots$ be independent, identically distributed, continuous random variables with a $N(2,9)$ distribution. Define $Y_{i}=(0.5)^{i} X_{i}, \quad i=$ $1,2, \ldots$. Also define $T_{n}$ and $A_{n}$ to be the sum and the average, respectively, of the terms $Y_{1}, Y_{2}, \ldots, Y_{n}$.

(a) Is $Y_{n}$ convergent in probability to 0 ?

(b) Is $T_{n}$ convergent in probability to 2 ?

(c) Is $A_{n}$ convergent in probability to 0 ?

(d) Is $T_{n}$ convergent in law to a $N(2,3)$ ? 


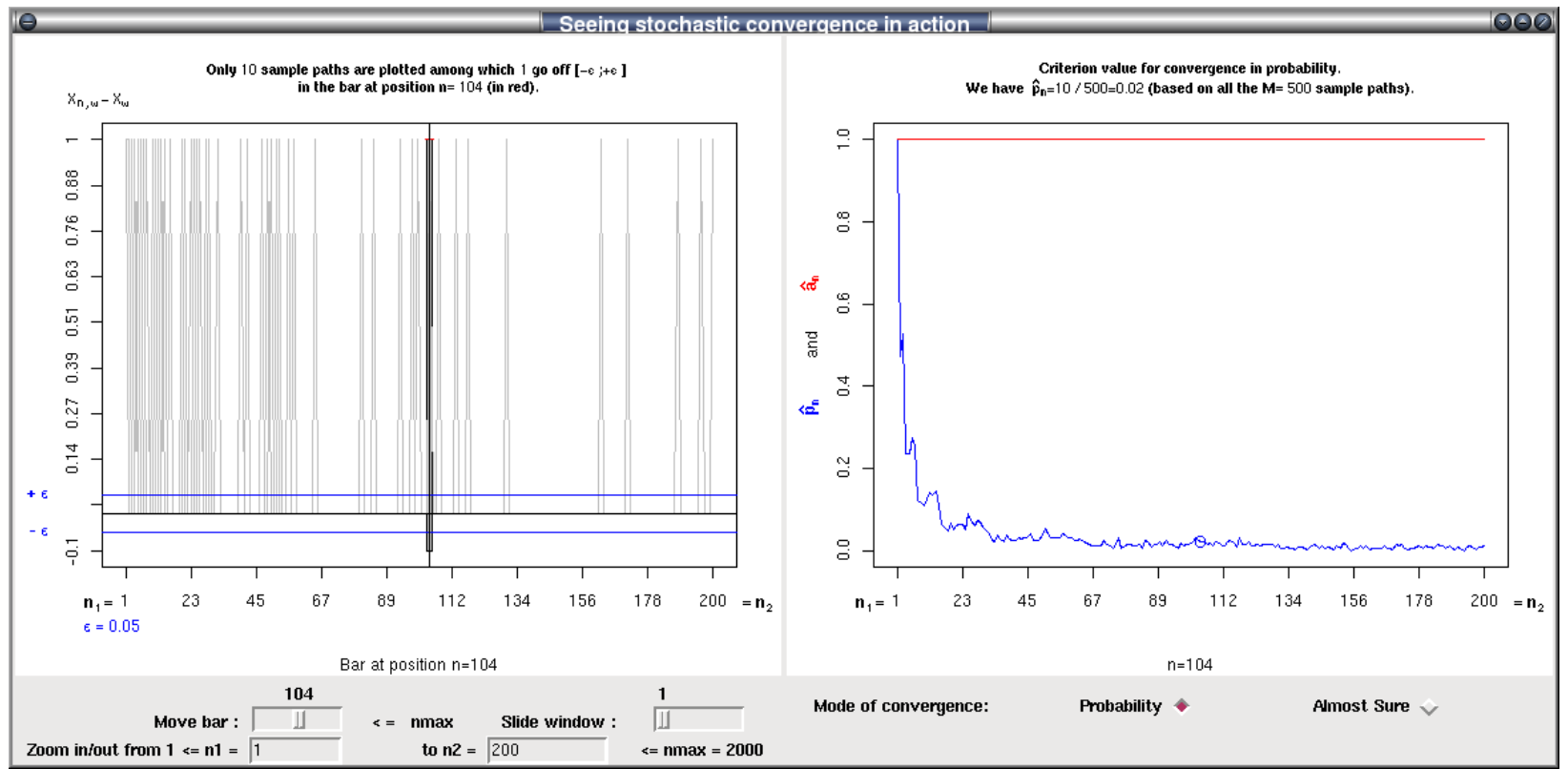

Figure 4: Seeing convergence in probability in action. On the left panel, we visualize only 10 sample paths among the $M=500$ simulated realizations of the sequence of random variables defined in exercise 1 . It can be seen that, among these ten, only one sample path go off $[-\epsilon,+\epsilon]$ in the bar at position $n=104$. On the right panel, we visualize the $\hat{p}_{n}$ values for each $n$ between 1 and 200 . We see that $\hat{p}_{n}$ goes towards 0 .

\section{Solution to problem 1:}

(a) We first define a random generator (called genYn) for the $Y_{i}^{\prime}$ 's then we call the function check. convergence.

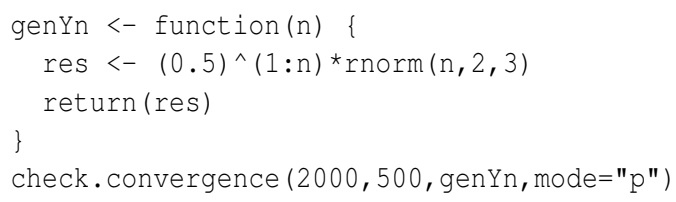

We can zoom in the left panel of Figure 5 (from $n_{1}=1$ to $n_{2}=10$ ) and see the 10 sample paths going rapidly inside the horizontal band $[-\epsilon,+\epsilon]$. Looking at the evolution of $\hat{p}_{n}$ towards 0 in the right panel, we can assume that $Y_{n}$ converges in probability towards 0 .

(b) We first define a random generator (called genTn) for the $\left(T_{i}-2\right)$ 's then we call the function check. convergence.

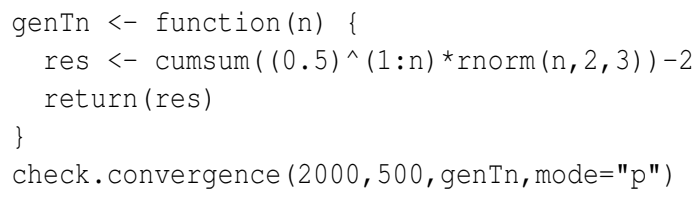

Each one of the sample paths rapidly evolve towards an horizontal asymptote, not the same for each sample path, and not contained inside the horizontal band $[-\epsilon,+\epsilon]$. Looking at the evolution of $\hat{p}_{n}$ in the right panel of Figure 6, we can assume that $T_{n}$ does not converge in probability towards 2 . (c) We first define a random generator (called genAn) for the $A_{i}$ 's then we call the function check. convergence.

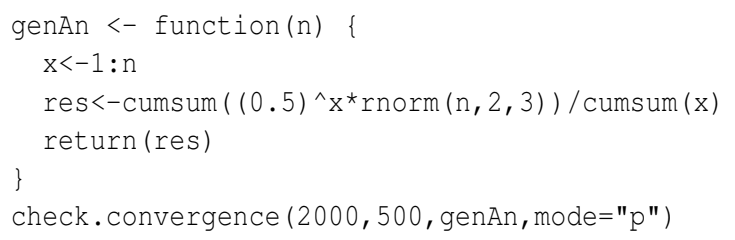

In this case, we can zoom in (from $n_{1}=1$ to $\left.n_{2}=50\right)$ to better see the sample paths which all end up inside the horizontal band $[-\epsilon,+\epsilon]$. Looking at the evolution of $\hat{p}_{n}$ towards 0 in the right panel of Figure 7 , we can assume that $A_{n}$ converges in probability towards 0 .

(d) We first define a random generator (called genTnL) for the $T_{i}$ 's then we call the function check. convergence.

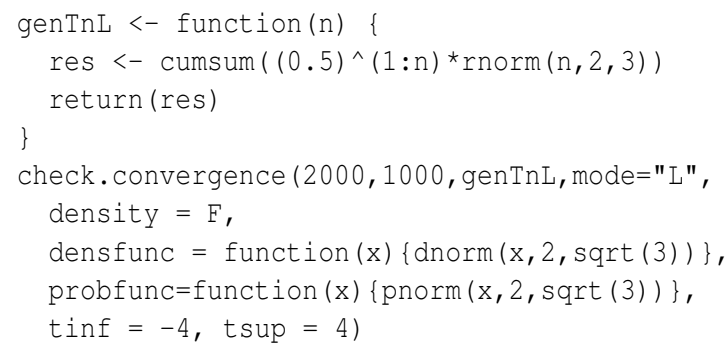

By definition, $X_{n} \stackrel{L}{\longrightarrow} X$ if and only if $l_{n}(t)=$ $\left|F_{n}(t)-F(t)\right| \underset{n \rightarrow \infty}{\longrightarrow} 0$, at all $t$ for which $F$ (the distribution function of $X$ ) is continuous, where 


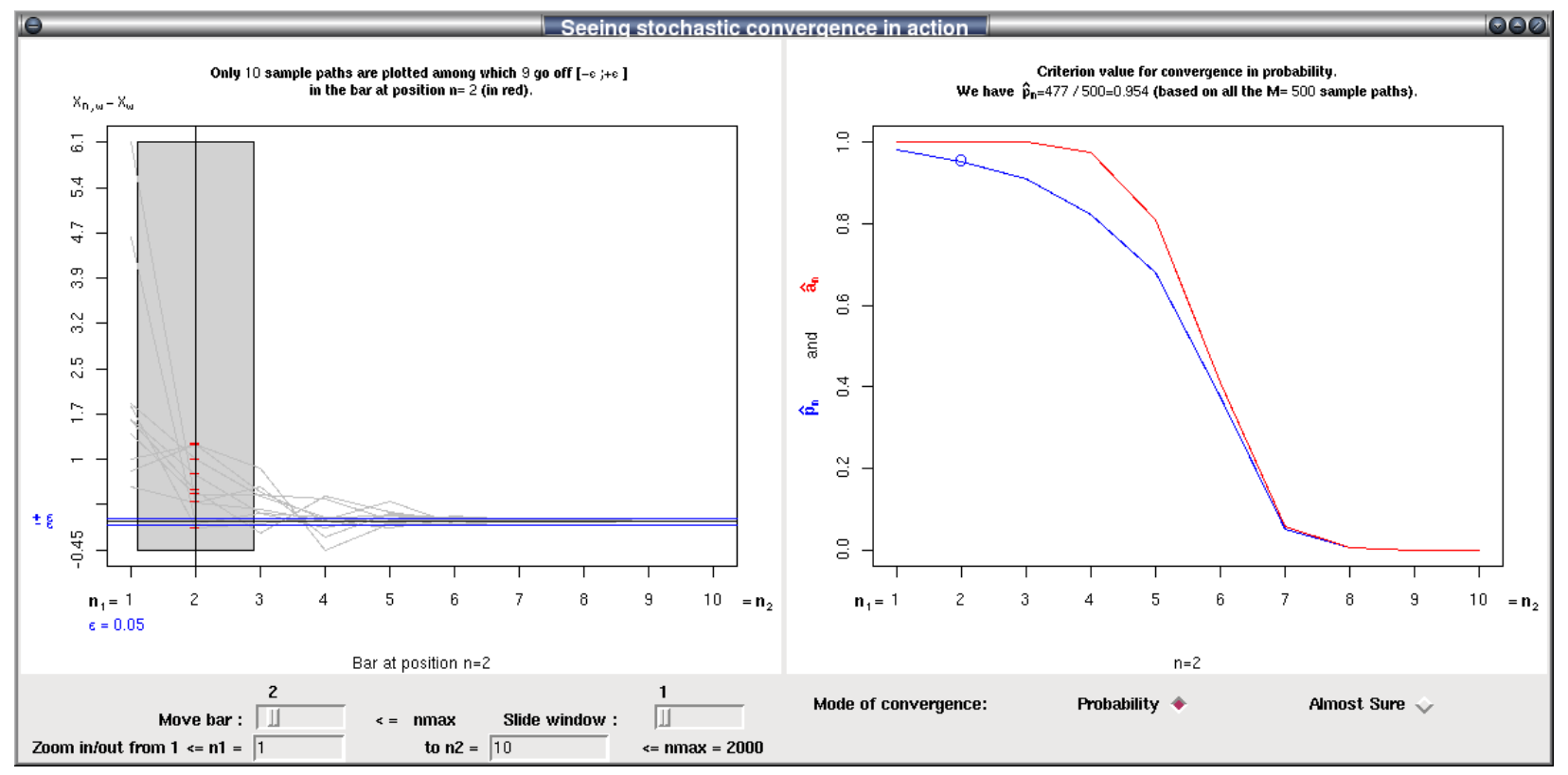

Figure 5: Investigating the convergence in probability towards 0 for the sequence of random variables $Y_{n}$ of Problem 1.(a). On the right panel, we see that $\hat{p}_{n}$ goes towards 0.

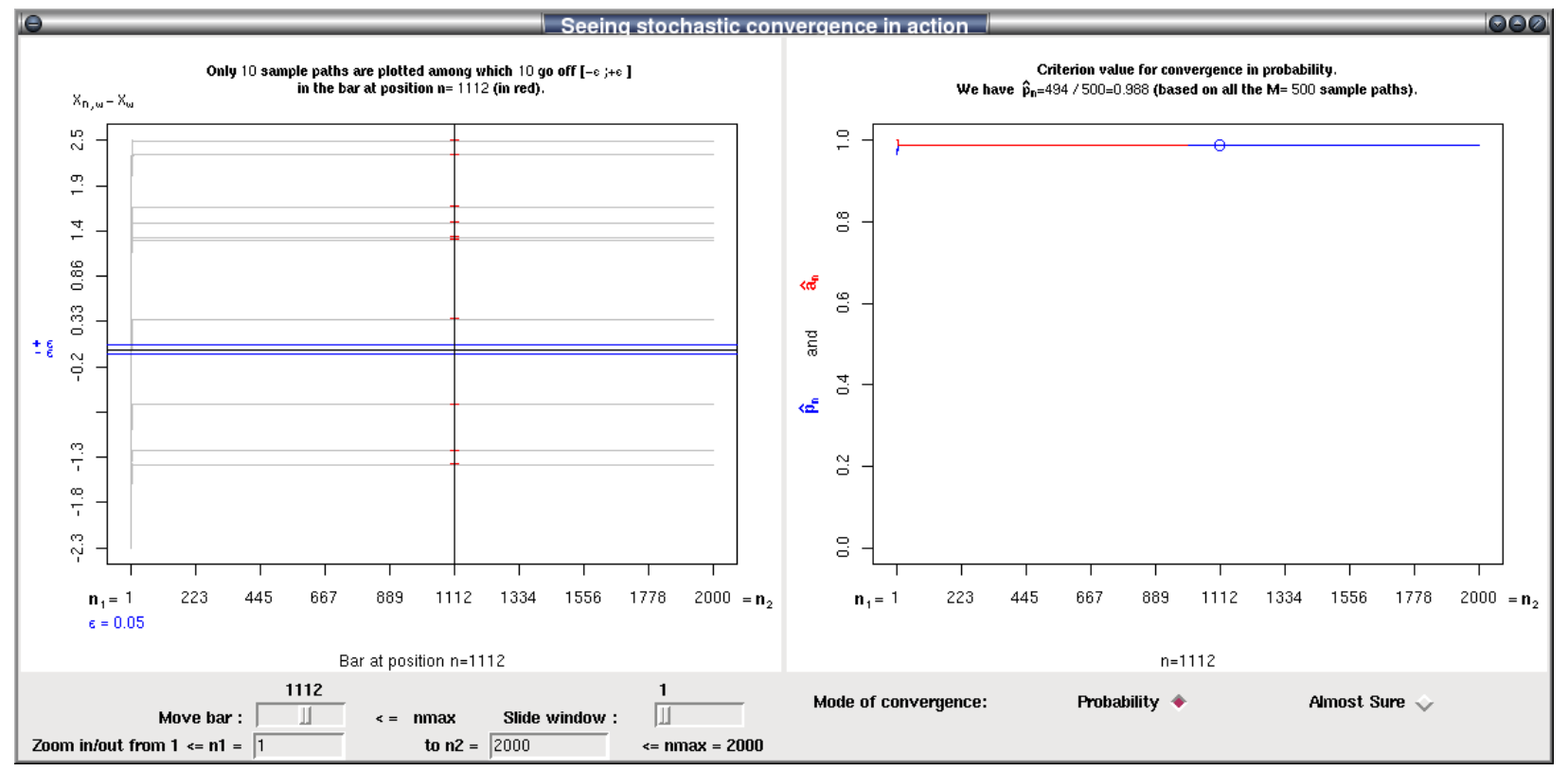

Figure 6: Investigating the convergence in probability towards 2 for the sequence of random variables $T_{n}$ of Problem 1.(b). On the right panel, we see that $\hat{p}_{n}$ equals 1. 


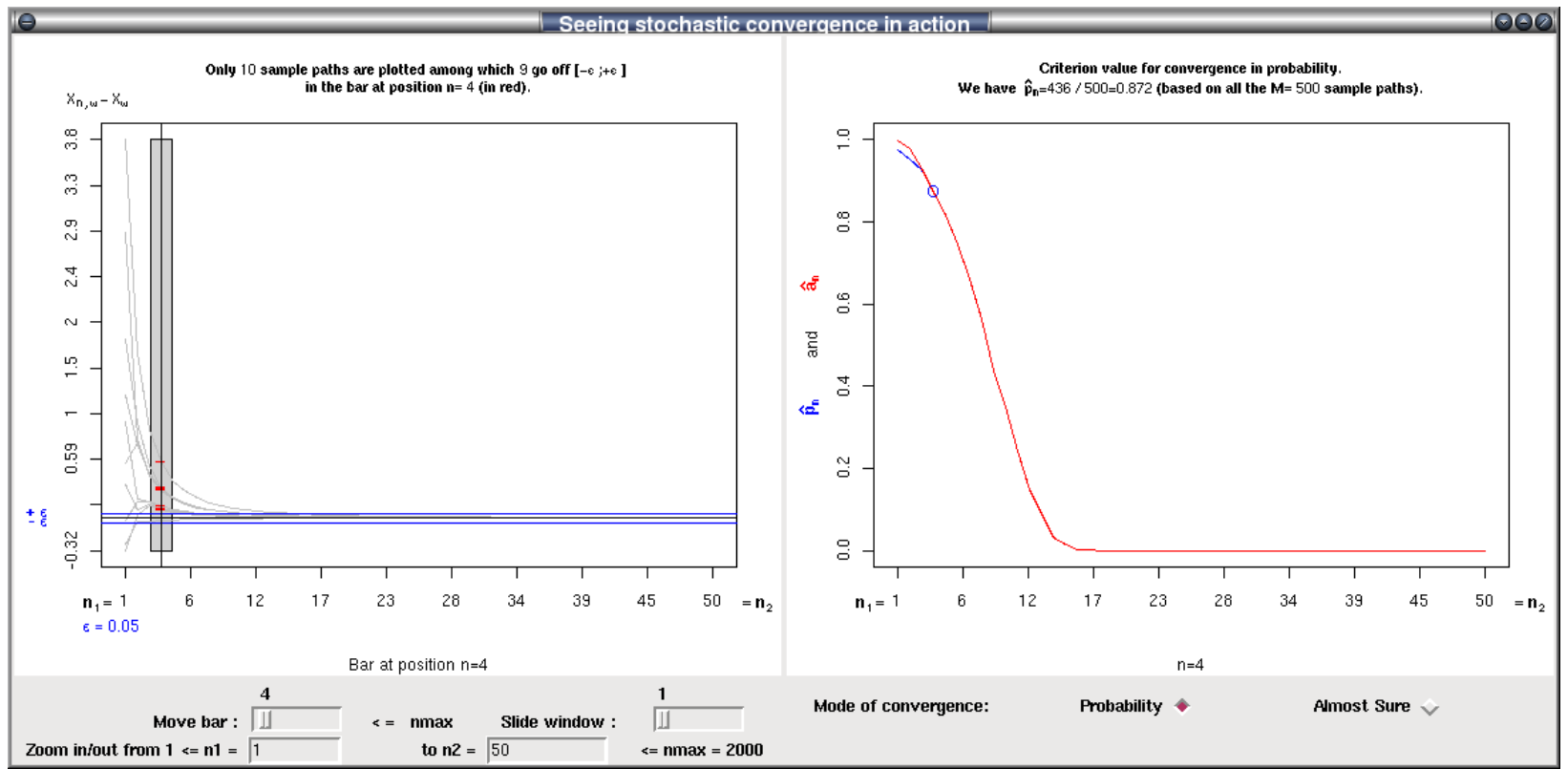

Figure 7: Investigating the convergence in probability towards 0 for the sequence of random variables $A_{n}$ of Problem 1.(c). On the right panel, we see that $\hat{p}_{n}$ goes towards 0 .
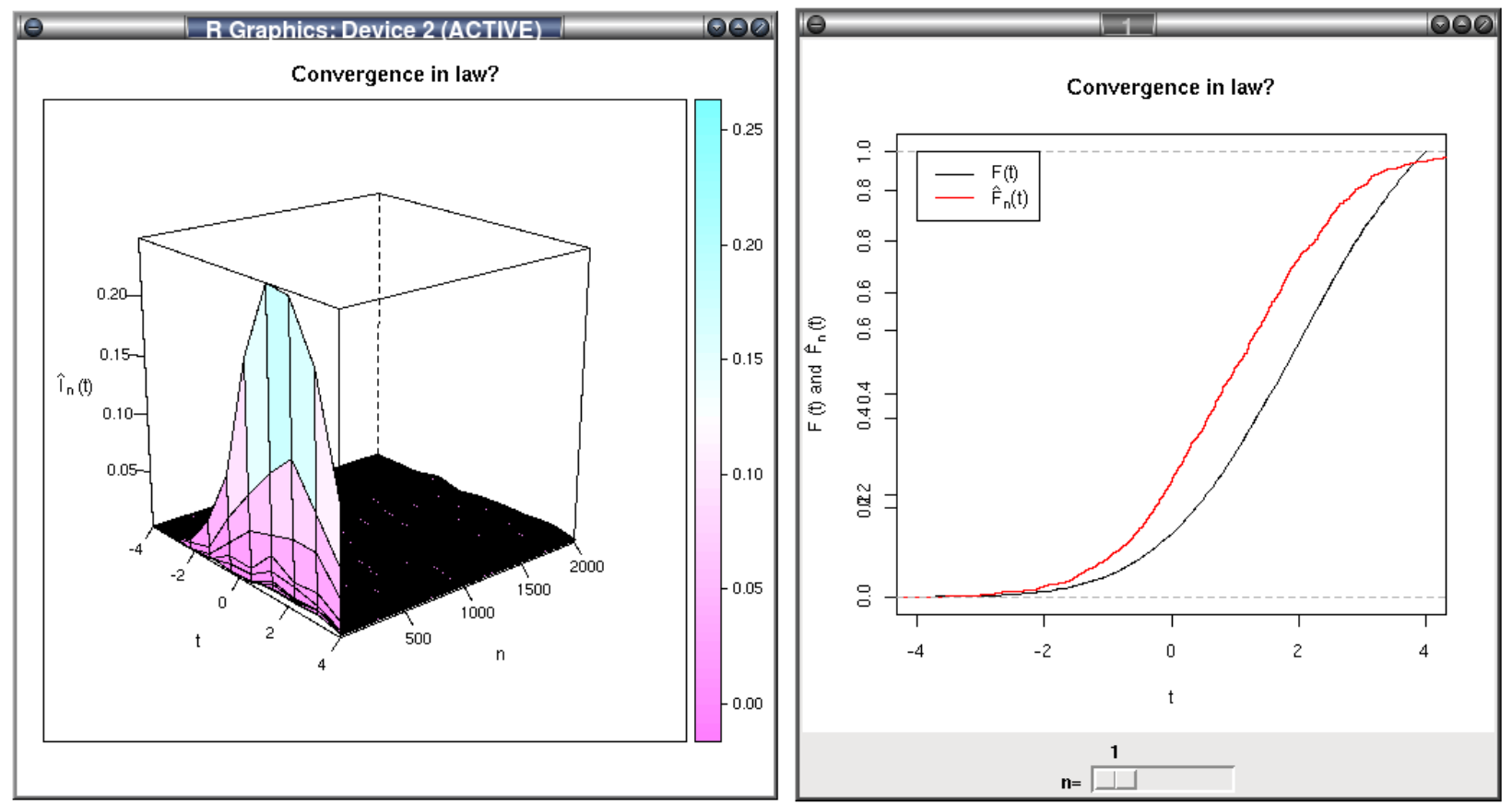

Figure 8: Investigating convergence in law. 
$F_{n}(t)$ is the distribution function of $X_{n}$. We define $\hat{F}_{n}(t)$ to be the empirical distribution function of $X_{n}$ (based on $M$ realizations of $X_{n}$ ) and $\hat{l}_{n}(t)=\left|\hat{F}_{n}(t)-F(t)\right|$.

We can move the slider (right part of Figure 8 ) and see that the red curve comes closer to the black one. Also, on the right you can see the tri-dimensional plot of $\left|\hat{F}_{n}(t)-F(t)\right|$ for $n=1, \ldots, n_{\max }=2000$ to see if gets closer to the zero horizontal plane. These plots suggest a convergence in distribution.

Problem 2: Let $X_{1}, X_{2}, \ldots$ be i.i.d. random variables with a uniform distribution on $[0,1]$. We define $M_{n}=$ $\max \left\{X_{1}, \ldots, X_{n}\right\}$.

(a) Show that $M_{n}$ converges in probability and almost surely to 1 .

(b) Show that $M_{n}$ converges in quadratic mean to 1.

\section{Solution to problem 2:}

We first define our random generator of the $\left(X_{i}-\right.$ $1)^{\prime}$ s.

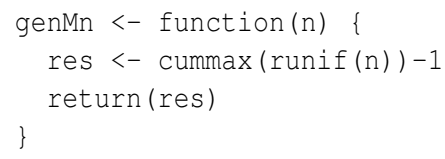

(a) We now call the check. convergence function.

check. convergence $(2000,500$, genMn, mode="p" )

Obviously, all the sample paths are strictly increasing towards 1 . Looking at the right panel of Figure 9 , we see $\hat{a}_{n}$ and $\hat{p}_{n}$ decreasing towards 0 . This makes us believe that we are contemplating a convergence almost surely and convergence in probability towards 1 .

(b) We now call the check. convergence function to investigate the quadratic mean convergence.

check. convergence $(2000,500$, genMn, mode $=" r ", r=2)$

By definition, $X_{n} \stackrel{r}{\longrightarrow} X$ if and only if $e_{n, r}=$ $E\left|X_{n}-X\right|^{r} \underset{n \rightarrow \infty}{\longrightarrow} 0$. We define, in an obvious fashion, $\hat{e}_{n, r}$ to be a Monte Carlo estimate of $e_{n, r}$, precisely defined in Lafaye de Micheaux and Liquet (2009).

Looking at Figure 10, one can expect $M_{n}$ to converge in quadratic mean towards 1 since $\hat{e}_{n, r}$ is decreasing towards 0 .

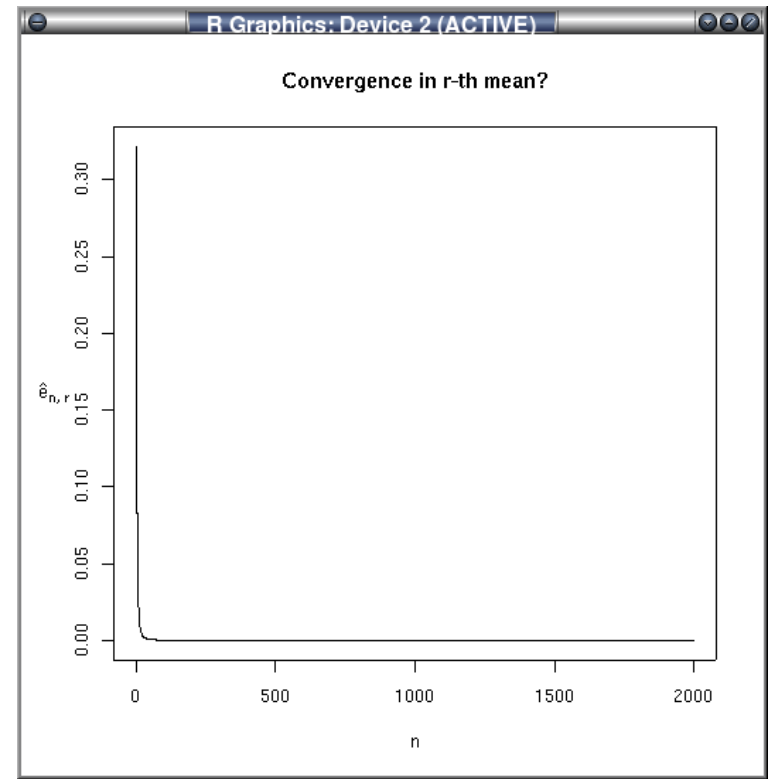

Figure 10: $\hat{p}_{n}$ and $\hat{a}_{n}$ going towards 0 .

\section{Conclusion}

We have described how this package can be used as interactive support for asymptotics courses. A few examples were given to show how to investigate almost sure convergence, convergence in probability, convergence in law, or in $r$-th mean.

\section{Bibliography}

P. Billingsley. Probability and Measure. Wiley, New York, 2nd edition, 1986.

G.R. Bryce, R. Gould, W. Notz and R. Peck, R. Curriculum guidelines for bachelor of science degrees in statistical science. The American Statistician, 55: 7-13, 2001.

B. Chance and A. Rossman. Investigating Statistical Concepts, Applications, and Methods. Duxbury, Belmont, 2006.

K.L. Chung. A Course in probability theory. Academic press, New York, 2nd edition, 1974.

P.K. Dunn. Three tools for interactively visualizing some distribution theory concepts. The American Statistician, 53:137-139, 1999.

T. Ferguson. A Course in Large Sample Theory. Chapman and Hall, 1996.

P. Lafaye de Micheaux and B. Liquet. Understanding convergence concepts: A visual-minded and graphical simulation-based approach. The American Statistician, 63(2):173-178, 2009.

E. Lehmann. Elements of Large-Sample Theory. Springer, 2nd edition, 2001. 


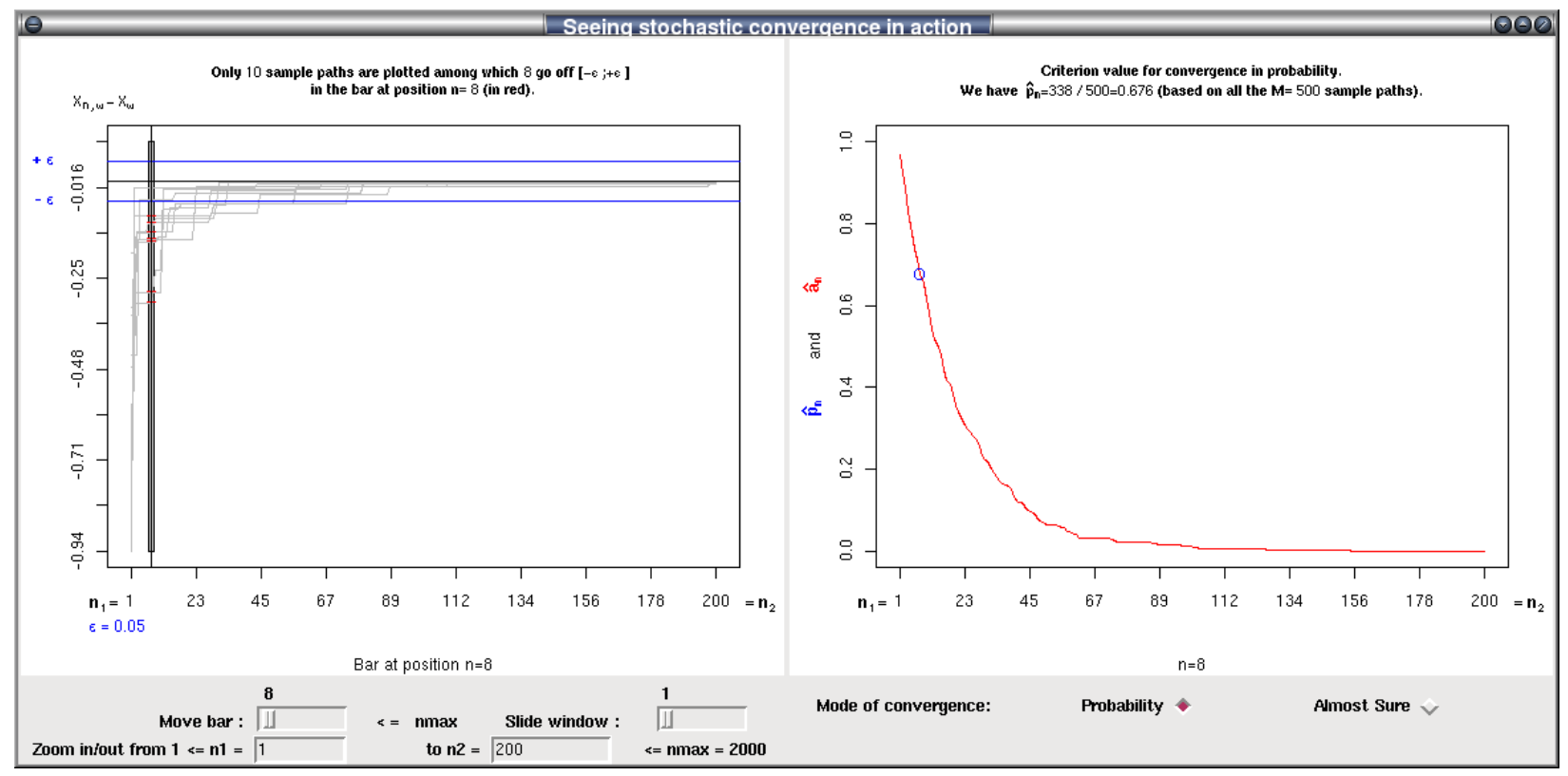

Figure 9: Investigating the convergence in probability and almost surely towards 1 for the sequence of random variables $M_{n}$ defined in Problem 2. On the right panel, we see the superimposed curves of $\hat{p}_{n}$ and $\hat{a}_{n}$ decreasing towards 0 .

M.G. Marasinghe, W.Q. Meeker, D. Cook and T. Shin. Using Graphics and Simulation to Teach Statistical Concepts. The American Statistician, 50:342-351, 1996.

J.D. Mills. Using computer simulation methods to teach statistics: A review of the literature. Journal of Statistics Education, 10(1), 2002. URL http: //www . amstat.org/publications/jse/ v10n1/mills.html.

R.J. Serfling. Approximation Theorems of Mathematical Statistics. Wiley, New York, 2002

J. Sethuraman. Review: Large-sample methods in statistics: An introduction with applications. Journal of The American Statistical Association, 90:384, 1995.
Pierre Lafaye de Micheaux

Département de Mathématiques et Statistique

Université de Montréal

Canada

http://www.biostatisticien.eu

lafayeddms.umontreal.ca

\section{Benoit Liquet}

INSERM U897, ISPED

University Bordeaux 2

France

Benoit.Liqueteisped.u-bordeaux2.fr 